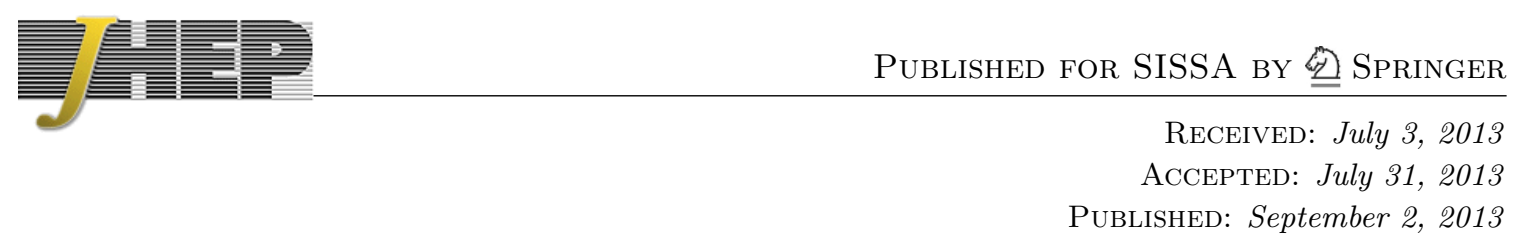

\title{
Isolating prompt photons with narrow cones
}

\author{
S. Catani, ${ }^{a}$ M. Fontannaz, ${ }^{b}$ J.Ph. Guillet ${ }^{c}$ and E. Pilon ${ }^{c}$ \\ ${ }^{a}$ INFN - Sezione di Firenze and Dipartimento di Fisica e Astronomia, \\ Università di Firenze, I-50019 Sesto Fiorentino, Florence, Italy \\ ${ }^{b}$ Laboratoire de Physique Théorique LPT, \\ UMR 8627 du CNRS, Université de Paris XI, F-91405 Orsay Cedex, France \\ ${ }^{c}$ LAPTh, Univ. de Savoie, CNRS, \\ B.P. 110, Annecy-Le-Vieux, F-74941, France \\ E-mail: catani@fi.infn.it, Michel.Fontannaz@th.u-psud.fr, \\ guillet@lapth.cnrs.fr, pilon@lapth.cnrs.fr
}

ABSTRACT: We discuss the isolation of prompt photons in hadronic collisions by means of narrow isolation cones and the QCD computation of the corresponding cross sections. We reconsider the occurence of large perturbative terms with logarithmic dependence on the cone size and their impact on the fragmentation scale dependence. We cure the apparent perturbative violation of unitarity for small cone sizes, which had been noticed earlier in next-to-leading-order (NLO) calculations, by resumming the leading logarithmic dependence on the cone size. We discuss possible implications regarding the implementation of some hollow cone variants of the cone criterion, which simulate the experimental difficulty to impose isolation inside the region filled by the electromagnetic shower that develops in the calorimeter.

KEywords: QCD Phenomenology, Hadronic Colliders

ARXIV EPRINT: 1306.6498 


\section{Contents}

1 Introduction 1

2 Origin of the logarithmic dependence on the cone size at the NLO 3

3 Multiparton collinear radiation and $\ln R$ dependence $\quad 6$

4 Isolated cross section and resummation of the $\ln R$ dependence $\quad 11$

5 Quantitative results 13

$\begin{array}{lll}6 & \text { Implications for a hollow-cone criterion } & 17\end{array}$

$\begin{array}{lll}7 \text { Conclusions } & 19\end{array}$

\section{Introduction}

The measurements of hard prompt photons by $\mathrm{TeV}$ collider experiments, such as those that were performed at the Fermilab Tevatron [1-6], RHIC [7], and those currently carried out at the CERN LHC [8-13], have long been recognized as important test of QCD quite sensitive to the gluon content inside the proton (see refs. [14, 15] for the recent revival of interest in prompt photon data to determine the gluon distribution function inside the proton). Prompt photons also provide Standard Model (SM) benchmarks useful to back up the understanding of the LHC detectors at the begining of the LHC era. Moreover, photon production and, especially, photon pair production ${ }^{1}$ [16-21] provide large SM backgrounds to signatures of various potential effects of new physics [22-26]. In this respect, one should distinguish the so-called prompt photons from what could be named secondary photons. Prompt photons directly take part in the hard partonic subprocess. Secondary photons instead originate from the decays of hadrons (e.g., $\pi^{0}$ and $\eta$ mesons) that are produced in the final (subsequent to the hard-scattering process) parton-to-hadron fragmentation stage of the hadronic collision. The huge yield of secondary photons at colliders overwhelms the production of prompt photons. This is even more true for signals of new physics involving photons with moderately large transverse momenta $\left(p_{T}\right)$, such as, for example, the search for the Higgs boson at LHC in the two-photon decay channel in the mass range $120-130 \mathrm{GeV}[27-30]$.

To reduce the background of secondary photons, collider experiments impose isolation cuts. The isolation cuts, which are optimized to the experimental setup, act on calorimetric deposits, tracks, discriminating shape observables and so forth, in a quite sophisticated

\footnotetext{
${ }^{1}$ References [1-13] and [16-21] include some of the most recent experimental results on isolated photons in hadron collisions and further references to previous experimental results.
} 
way. Such criteria can be taken into account in full-fledged event simulations but not in calculations performed at the parton level only. A customary basic requirement that can be implemented in parton level calculations is transverse-energy isolation. One considers the photon candidate and the direction of its momentum $p^{\gamma}$, as specified by the rapidity and azimuthal-angle variables $y_{\gamma}$ and $\phi_{\gamma}$ (rapidities, azimuthal angles and transverse energies are defined in a reference frame where the momenta of the two colliding hadrons are back-to-back). Around the direction of the photon candidate, one draws a cone $\mathcal{C}_{\gamma}(R)$ of aperture $R$ in $\{y, \phi\}$ space and considers the hadrons (each hadron with momentum $p_{k}$ and corresponding transverse energy $E_{T}^{(k)}$ ) inside the cone:

$$
\text { hadron } k \in \mathcal{C}_{\gamma}(R) \Leftrightarrow \sqrt{\left(y_{k}-y_{\gamma}\right)^{2}+\left(\phi_{k}-\phi_{\gamma}\right)^{2}} \leq R \text {. }
$$

Then one requires that the total amount of hadronic transverse energy inside this cone is smaller than a maximum amount $E_{T}^{\text {iso }}$ :

$$
\sum_{k \in \mathcal{C}_{\gamma}(R)} E_{T}^{(k)}<E_{T}^{\text {iso }}
$$

Recent improvements of this isolation criterion recommend to first subtract the contribution coming from the underlying event and from pile-up, before the criterion is applied; the contribution to be subtracted is assessed from the mini-jet activity away from the direction of the photon candidate [31,32]. Other criteria at variance with that of eqs. (1.1) and (1.2) have also been proposed [33-37]. All these isolation criteria have impact on the selected sample of prompt photons. As described for instance in refs. [38, 39], prompt photons may be schematically viewed as produced by two mechanisms: the "direct" (D) mechanism, in which the photon is produced directly at high $p_{T}$ by hard scattering, and the "fragmentation" (F) mechanism, in which the photon originates from the (essentially collinear) fragmentation of a high- $p_{T}$ coloured parton primarily produced by hard scattering. The isolation criteria have impact on both "direct" and "fragmentation" photons. In particular, the production rate through the fragmentation mechanism is strongly reduced by the isolation, since the "fragmentation" photon is generally produced inside a large- $p_{T}$ jet of hadrons (unless the photon carries a major fraction of its parent parton's transverse momentum).

QCD radiative corrections for isolated prompt-photon production at hadron colliders have been computed in the literature. The next-to-leading order (NLO) QCD corrections to single-inclusive photon production were computed in ref. [40] (using cone isolation in the small- $R$ approximation) and ref. [38] (for any infrared-safe isolation criteria). Diphoton production has been computed at the NLO [39, 41], including NLO corrections [42] to the gluon fusion channel, and at the next-to-next-to-leading order (NNLO) [43] (using the isolation criterion of ref. [35]). The NLO calculation of 'photon plus one jet' was performed in ref. [44]. Diphoton production in association with one jet [45] and two jets [46] has been computed at the NLO by using the isolation criterion of ref. [35]. The NLO calculation of 'diphoton plus one jet' for general isolation criteria has been performed recently [47]. Higher-order QCD contributions due to soft gluons [48-50], high-energy logarithmic corrections [51-53], and parton shower effects [54-56] have also been studied. 
In the present article we will focus on the "standard cone criterion" defined by eqs. (1.1) and (1.2), and on its implementation in QCD calculations at partonic level. We will also discuss some implications for the implementation of a 'two cone' criterion that aims at simulating a poorer isolation around the electromagnetic cluster of photon candidates in some experimental configurations. In ref. [38] we studied the standard cone criterion, and we presented the calculation of isolated-photon cross sections at the NLO of the perturbative expansion in powers of the QCD coupling $\alpha_{s}$. In particular, we studied the dependence of the cross section on the size $R$ of the isolation cone. Considering small values of $R$ (typically $R \lesssim 0.1$ ), we noticed [38] a violation of unitarity of the NLO result, since the NLO isolated cross section becomes larger than the NLO inclusive (i.e., without isolation) cross section. Therefore, at small values of $R$, the NLO result is certainly unphysical. Moreover, this finding shed doubts on the reliability of the NLO QCD prediction also at moderately-small values of $R(R \sim 0.4-0.3)$ that are actually used in experiments. The purpose of this article is to trace back this misbehaviour of the NLO result and to cure it.

The paper is organized as follows. We detail how the $R$ dependence, which is dominantly logarithmic at small $R$ order by order in perturbation theory, appears in both the NLO calculation (section 2) and at higher-order levels (section 3 ). We point out how the isolation constraint on transverse energy causes a mismatch in the $R$ dependence produced by parton radiation inside and outside the isolation cone. This mismatch produces the observed violation of unitarity in the NLO calculation at small values of $R$, and it makes an all-order resummation of the ensuing $\ln R$ terms mandatory. In section 4 we discuss the implementation of resummation to leading logarithmic (LL) accuracy for the standard cone isolation criterion. Then, in section 5, we present numerical results at Tevatron and LHC energies, and we explicitly show how LL resummation removes the unphysical behaviour of the NLO calculation at small values of $R$. In section 6 we discuss some implications of these results for the implementation of a criterion based on a hollow cone, which (very crudely) mimics the difficulty to experimentally implement isolation in the solid angle filled by the electromagnetic cluster of a hard photon in a detector. A brief summary is presented in section 7 .

\section{Origin of the logarithmic dependence on the cone size at the NLO}

We consider the isolation criterion in eqs. (1.1) and (1.2) and the ensuing small- $R$ behaviour of the isolated-photon cross section at the NLO. To identify the origin of the logarithmic dependence on $R$, we briefly recall the results obtained in refs. $[38,40]$ on the calculation of the higher-order ( $\mathrm{HO}$ ) contribution to the Born level cross section.

We start with the contribution where the photon is accompanied by collinear parton radiation inside a cone of radius $R$ (see figure 1). This part of the $\mathrm{HO}$ correction to the Born level direct (D) cross section leads to the NLO fragmentation (F) contribution. Using dimensional regularization in $d=4-2 \epsilon$ space-time dimensions, the differential cross section with respect to the transverse momentum $\vec{p}_{T}^{\gamma}$ and the rapidity (or, equivalently, pseudorapidity) $\eta^{\gamma}$ of the photon is (we refer the reader to ref. [38] for more details about 


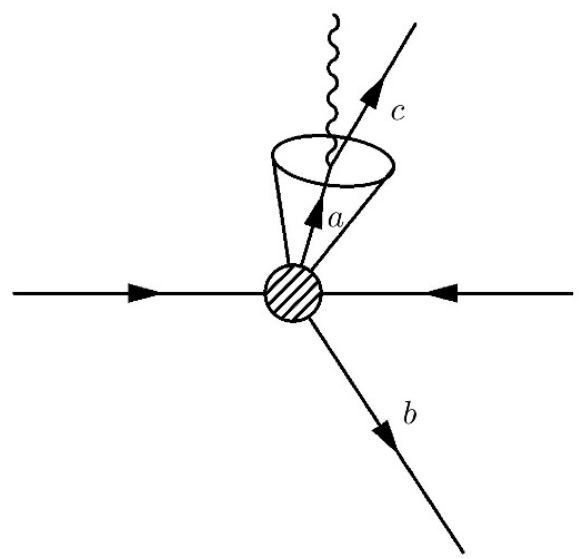

Figure 1. A pictorial representation of the NLO contribution from final-state collinear radiation inside the isolation cone.

the notation)

$$
\begin{aligned}
& \left.\frac{d \sigma}{d \vec{p}_{T}^{\gamma} d \eta \gamma}\right|_{\text {coll inside cone }} ^{(H O)} \\
& =\left(\frac{\alpha_{s}}{\pi}\right)^{2} \int_{z_{\min }}^{1} \frac{d \sigma^{\text {Born }}}{d \vec{p}_{T}^{a} d \eta^{a} d \eta^{b}}(A+B \rightarrow a+b) d \eta^{b} \frac{d z}{z^{2}} e_{a}^{2} \frac{\alpha}{2 \pi} \\
& \quad\left\{P_{q \gamma}(z)\left[-\frac{(4 \pi)^{\epsilon}}{\epsilon} \frac{\Gamma(1-\epsilon)}{\Gamma(1-2 \epsilon)}+\ln \left(\frac{M_{F}^{2}}{\mu_{\mathrm{reg}}^{2}}\right)+\ln \left(\frac{R^{2}\left(p_{T}^{\gamma}\right)^{2}}{M_{F}^{2}}\right)+\ln \left((1-z)^{2}\right)\right]+z\right\} .
\end{aligned}
$$

The factor

$$
\frac{d \sigma^{\text {Born }}}{d \vec{p}_{T}^{a} d \eta^{a} d \eta^{b}}(A+B \rightarrow a+b)
$$

is the Born level cross section (the overall power of $\alpha_{s}$ is not included in $d \sigma^{\text {Born }}$ and it is explicitly denoted in eq. (2.1)) of the reaction hadron $A+$ hadron $B \rightarrow$ parton $a+$ parton $b$. In the NLO contribution of eq. (2.1), the fragmenting parton $a$ and the collinear parton $c$ are either quarks or antiquarks.

To obtain the expression on the right-hand side of eq. (2.1), the integration over the angular phase space of parton $c$ has been restricted to lie inside the cone and, moreover, we have used the small-cone approximation, thus neglecting terms of $\mathcal{O}\left(R^{2}\right)$. The integration over the momentum fraction $z=p_{T}^{\gamma} / p_{T}^{a}$ is bounded by $z_{\min }$, which is fixed by the photon kinematics and the centre-of-mass energy $\sqrt{S}$ of the two colliding hadrons. The dimensional regularization scale is denoted by $\mu_{\mathrm{reg}}$, and for later convenience we have introduced the auxiliary factorization scale $M_{F}$ (the right-hand side of eq. (2.1) is actually independent of $M_{F}$ ).

The first two terms in the curly bracket of eq. (2.1) correspond to (the $\epsilon$-expansion of) the bare photon fragmentation function $D_{a}^{\gamma \text { bare }}$ in the customary $\overline{\mathrm{MS}}$ factorization scheme,

$$
D_{a}^{\gamma \text { bare }}\left(z, M_{F}, \epsilon\right)=-\frac{1}{\epsilon} \frac{\Gamma(1-\epsilon)}{\Gamma(1-2 \epsilon)}\left(\frac{4 \pi \mu_{\mathrm{reg}}^{2}}{M_{F}^{2}}\right)^{\epsilon} K_{a}^{(0)}(z)
$$


with

$$
K_{a}^{(0)}(z)=\frac{\alpha}{2 \pi} e_{a}^{2} \frac{1+(1-z)^{2}}{z} \equiv \frac{\alpha}{2 \pi} e_{a}^{2} P_{q \gamma}(z)
$$

and where $\alpha$ is the fine structure constant and $e_{a}$ is the electric charge of the parton $a$ $\left(K_{g}^{(0)}(z)=0\right.$ since $\left.e_{g}=0\right)$. Within the $\overline{\mathrm{MS}}$ factorization scheme, the other terms in the curly bracket of eq. (2.1) are considered as HO corrections to the direct cross section coming from parton radiation inside the cone. The proper treatment of the collinear $1 / \epsilon$ singularity to all orders (as discussed in details, e.g., in refs. [57, 58]) leads to the introduction of the all-order fragmentation functions $D_{a}^{\gamma}\left(z, M_{F}\right)$. These fragmentation functions obey the following inhomogeneous evolution equations:

$$
M_{F}^{2} \frac{\partial D_{a}^{\gamma}}{\partial M_{F}^{2}}=K_{a}+\sum_{b} P_{b a} \otimes D_{b}^{\gamma},
$$

where the symbol $\otimes$ denotes the following convolution:

$$
(f \otimes g)(z)=\int_{0}^{1} d u \int_{0}^{1} d v f(u) g(v) \delta(u v-z) .
$$

The all-order functions $K_{a}(z)$ are the inhomogeneous kernels for the collinear splitting 'parton $a$ to photon',

$$
K_{a}(z)=K_{a}^{(0)}(z)+\frac{\alpha_{s}}{2 \pi} K_{a}^{(1)}(z)+\cdots,
$$

where the leading order (LO) term $K_{a}^{(0)}(z)$ is given in eq. (2.3). The all-order functions $P_{a b}(z)$ are the usual Dokshitzer-Gribov-Lipatov-Altarelli-Parisi (DGLAP) time-like splitting kernels,

$$
P_{b a}(z)=\frac{\alpha_{s}}{2 \pi} P_{b a}^{(0)}(z)+\left(\frac{\alpha_{s}}{2 \pi}\right)^{2} P_{b a}^{(1)}(z)+\cdots,
$$

where $P_{b a}^{(0)}(z)$ is the LO term, $P_{b a}^{(1)}(z)$ is the NLO term and so forth. A thorough discussion of the evolution equation (2.4) and its solutions with appropriate boundary conditions can be found in ref. [57].

The contribution coming from the integration over the phase space region where parton $c$ is outside the cone contains a term proportional to $\ln (1 / R)$. When no isolation is imposed on the collinear debris that accompanies the photon, the $\ln (1 / R)$ dependence from outside the cone completely cancels against the $\ln R$ term of eq. (2.1). On the contrary, in the case of the isolated cross section, when the parton $c$ lies inside the cone the isolation requirement of eq. (1.2) leads to the constraint $p_{T}^{c} \leq E_{T}^{\text {iso }}\left(p_{T}^{c}=(1-z) p_{T}^{a}=(1-z) p_{T}^{\gamma} / z\right)$, which restricts the integration range over $z$ in eq. (2.1) to the following region:

$$
z \geq z_{\text {cut }} \equiv \frac{1}{1+\varepsilon} \quad, \quad \varepsilon \equiv \frac{E_{T}^{\text {iso }}}{p_{T}^{\gamma}}
$$

This restriction produces a mismatch of the $\ln R$ dependences from inside vs. outside the cone, and this leads to a net $\ln R$ dependence in the isolated cross section. At the NLO this $\ln R$ dependence is given by the term

$$
-\left(\frac{\alpha_{s}}{\pi}\right)^{2} \int_{z_{\min }}^{z_{\mathrm{cut}}} \frac{d \sigma^{\text {Born }}}{d \vec{p}_{T}^{a} d \eta^{a} d \eta^{b}} d \eta^{b} \frac{d z}{z^{2}} K_{a}^{(0)}(z) \ln \left(R^{2}\right)
$$




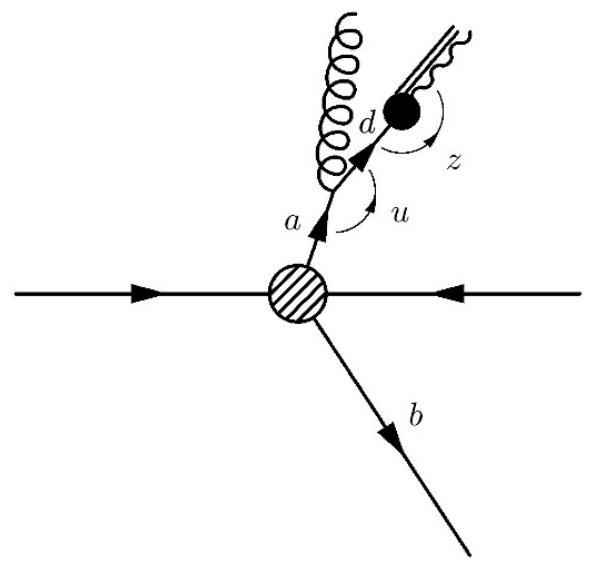

Figure 2. A HO correction to the fragmentation contribution.

that blows up towards $+\infty$ when $R \rightarrow 0$. This unbounded increase of the NLO isolated cross section leads to the violation of unitarity that was observed in ref. [38]. As pointed out in ref. [38], this unphysical effect is an artefact of the fixed-order truncation of the QCD perturbative series: an all-order summation in $\alpha_{s}$ of the $\ln R$ terms should cure the pathological behaviour induced at the NLO by the contribution in eq. (2.6).

We have so far discussed the HO correction to the Born level direct cross section. An analogous discussion applies to the HO correction to the Born level fragmentation component of the cross section (figure 2). In this case the photon production process proceeds through the fragmentation function $D_{d}^{\gamma}$ (which contains a perturbative and a non-perturbative component) of a parton $d$. The effects of collinear parton radiation inside and outside the cone around the parton $d$, and the ensuing mismatch of the $\ln R$ dependence in the NLO isolated cross section produce a contribution that is analogous to the term in eq. (2.6). The main difference with respect to eq. (2.6) is that the factor $K_{a}^{(0)}(z) \ln \left(R^{2}\right)$ is replaced by the factor

$$
\frac{\alpha_{s}}{2 \pi} P_{d a}^{(0)}(z / x) \ln \left(R^{2}\right)
$$

that is then convoluted with the fragmentation function $D_{d}^{\gamma}\left(x, M_{F}\right)$. At fixed values of $M_{F}$, this $\ln R$ contribution to the fragmentation component of the NLO isolated cross section also blows up towards $+\infty$ when $R \rightarrow 0$. Therefore, the violation of unitarity produced by the NLO direct contribution is not removed by the NLO fragmentation contribution. The all-order summation of the $\ln R$ terms should cure the pathological behaviour observed at the NLO in both the direct and fragmentation components of the isolated cross section.

\section{Multiparton collinear radiation and $\ln R$ dependence}

The collinear-radiation spectrum of a single parton that is emitted inside a small cone and just outside of it produces a $\ln R$ contribution to the NLO cross section. This effect has been briefly recalled in section 2 . In the present section, we consider the effect of multiple collinear radiation, and we illustrate how the $\ln R$ contributions arise at all orders in QCD 
perturbation theory. The discussion of this section sets the stage for the resummation of the $\ln R$ dependence of the isolated cross section, which is considered in section 4 .

The discussion is more conveniently formulated by taking Mellin moments of the relevant expressions. In the following we use a shorthand notation. For instance, the $n$-moment $\sigma(n)$ of the differential cross section $\frac{d \sigma}{d p_{T}^{\gamma} d \eta^{\gamma}}$ is defined as follows:

$$
\sigma(n)=\int_{0}^{1} d x_{T}^{\gamma}\left(x_{T}^{\gamma}\right)^{n-1} \frac{d \sigma}{d p_{T}^{\gamma} d \eta^{\gamma}}, \quad x_{T}^{\gamma} \equiv \frac{2 p_{T}^{\gamma}}{\sqrt{S}}
$$

and eq. (2.1) can be written as

$$
\sigma^{(H O)}(n)=\left(\frac{\alpha_{s}}{\pi}\right)^{2} \sigma_{a}^{\text {Born }}(n)\left[D_{a}^{\gamma \text { bare }}\left(n, M_{F}, \epsilon\right)+K_{a}^{(0)}(n) \ln \left(\frac{R^{2}\left(p_{T}^{\gamma}\right)^{2}}{M_{F}^{2}}\right)+t_{a}(n)\right],
$$

with

$$
\begin{aligned}
D_{a}^{\gamma \text { bare }}\left(n, M_{F}, \varepsilon\right) & =\int_{0}^{1} d z z^{n-1} D_{a}^{\gamma \text { bare }}\left(z, M_{F}, \epsilon\right) \\
& =-\frac{1}{\epsilon} \frac{\Gamma(1-\epsilon)}{\Gamma(1-2 \epsilon)}\left(\frac{4 \pi \mu_{\mathrm{reg}}^{2}}{M_{F}^{2}}\right)^{\epsilon} K_{a}^{(0)}(n),
\end{aligned}
$$

and where we have introduced

$$
\begin{aligned}
K_{a}^{(0)}(n) & =\frac{\alpha}{2 \pi} e_{a}^{2} \int_{0}^{1} d z z^{n-1} P_{q \gamma}(z) \\
t_{a}(n) & =\frac{\alpha}{2 \pi} e_{a}^{2} \int_{0}^{1} d z z^{n-1}\left[\frac{1+(1-z)^{2}}{z} \ln \left((1-z)^{2}\right)+z\right] .
\end{aligned}
$$

The all-order evolution equation (2.4) written in Mellin moments leads to a solution in closed form. We write it here explicitly for the flavour non-singlet (NS) component ${ }^{2}$ (and we drop all flavour indices, for the sake of simplicity):

$$
D\left(n, M_{F}\right)=\int_{M_{0}^{2}}^{M_{F}^{2}} \frac{d k^{2}}{k^{2}} K(n) e^{\int_{k^{2}}^{M_{F}^{2}} \frac{d k^{\prime 2}}{k^{\prime 2}} P(n)}+e^{\int_{M_{0}^{2}}^{M_{F}^{2}} \frac{d k^{\prime 2}}{k^{\prime 2}} P(n)} D\left(n, M_{0}\right),
$$

where the splitting kernels $K(n)$ and $P(n)$ are those of the NS case. Considering the first term in the right-hand side of eq. (3.6) and taking the lower bound of the $k^{2}$-integral equal to zero leads to collinear divergences, whose dimensional regularization produces $1 / \epsilon$ poles as in eqs. (2.2) and (3.3). Following the procedure described in refs. [57, 58], in the expression (3.6) dimensional regularization has been replaced by the cut-off $M_{0}$, which corresponds to the boundary between the perturbative and non-perturbative domains. Expanding the first term in the right-hand side of eq. (3.6), we indeed recover the perturbative expression of zeroth order in $\alpha_{s}$ of the NS fragmentation function,

$$
D^{(L O)}\left(n, M_{F}\right)=2\left(e_{a}^{2}-\left\langle e_{a}^{2}\right\rangle\right) \frac{\alpha}{2 \pi} P_{\gamma q}(n) \ln \left(\frac{M_{F}^{2}}{M_{0}^{2}}\right) .
$$

\footnotetext{
${ }^{2}$ In the NS component the factor $e_{a}^{2}$ in $K_{q}^{(0)}$ has to be replaced by $2\left(e_{a}^{2}-\left\langle e_{a}^{2}\right\rangle\right)$, where $\left\langle e_{a}^{2}\right\rangle=\frac{1}{N_{f}} \sum_{b=1}^{N_{f}} e_{b}^{2}$.
} 
The second term in the right-hand side of eq. (3.6) is thus proportional to $D\left(n, M_{0}\right)$, which is the non-perturbative contribution to the fragmentation function at the cut-off scale $M_{0}$.

The all-order perturbative structure of the cross section in eq. (3.1) follows from the QCD factorization theorem of collinear singularities. Considering the NS contribution to the cross section, the factorization theorem allows us to write

$$
\sigma(n)=\sigma_{F}\left(n, M_{F}\right) D\left(n, M_{F}\right)+\sigma_{D}\left(n, M_{F}\right)
$$

where the first term on the right-hand side is the fragmentation component, and the second term is the direct component (this separation in two components depends on the factorization scale $\left.M_{F}\right)$. The partonic subprocess cross sections $\sigma_{F}\left(n, M_{F}\right)$ and $\sigma_{D}\left(n, M_{F}\right)$ have an expansion in $\alpha_{s}$ of the form

$$
\begin{aligned}
& \sigma_{F}\left(n, M_{F}\right)=\left(\frac{\alpha_{s}}{\pi}\right)^{2} \sigma_{F}^{\text {Born }}(n)+\sigma_{F}^{(1)}\left(n, M_{F}\right)+\cdots, \\
& \sigma_{D}\left(n, M_{F}\right)=\frac{\alpha_{s}}{\pi} \sigma_{D}^{\text {Born }}(n)+\sigma_{D}^{(1)}\left(n, M_{F}\right)+\cdots,
\end{aligned}
$$

with $\sigma_{F}^{(k)} \sim \mathcal{O}\left(\alpha_{s}^{2+k}\right)$ and $\sigma_{D}^{(k)} \sim \mathcal{O}\left(\alpha_{s}^{1+k}\right)$.

We are interested in computing and resumming the $\ln R$-enhanced perturbative terms that arise in the presence of an isolation cone of small size $R$. Before studying the effect of isolation, we first consider the non-isolated cross section to all orders. We partition the available phase space by introducing a cone of size $R$ around the photon, and we consider the effect of QCD radiation inside and outside this cone. This partition artificially splits the cross section into terms that separately and explicitly depend on $R$, although the complete cross section is independent of $R$. To compute the all-order $\ln R$-dependence produced by this splitting, we exploit the basic physical picture that underlies the factorization structure of eq. (3.8). Indeed, the photon fragmentation function originates from the resummation of multiparton collinear radiation that is produced around the photon direction. Roughly speaking, the fragmentation function $D\left(n, M_{F}\right)$ embodies parton radiation inside a cone whose radius is of the order of $M_{F} / p_{T}^{\gamma}$. Moreover, the all-order evolution equation (2.4) follows from the angular ordered structure of multiple QCD radiation around the photon direction. Thus, the solution in eq. (3.6) of the evolution equation corresponds to the resummation of QCD radiation from small angles (of the order of $M_{0} / p_{T}^{\gamma}$ ) up to large angles (of the order of $M_{F} / p_{T}^{\gamma}$ ) with respect to the photon direction.

Owing to the physical picture that we have just described, we can easily evaluate the $\ln R$ terms that are produced by partitioning the phase space through the introduction of a cone of small size $R$. We consider the fragmentation function $D(n, \mathcal{M})$ where the scale $\mathcal{M}$, which is of the order of $p_{T}^{\gamma}$ (the precise value of $\mathcal{M}$ does not affect the following discussion at the level of the LL approximation), corresponds to the phase space region in which the collinear approximation neglecting the virtuality $p_{a}^{2}$ of parton $a$, cf. figure 2 , in the cross section of the $2 \rightarrow 2$ subprocess holds. The choice $\mathcal{M} \sim p_{T}^{\gamma}$ implies that $D(n, \mathcal{M})$ embodies collinear radiation up to very large angles with the respect to the photon direction. Therefore we can introduce the effect of the small-size cone by splitting $D(n, \mathcal{M})$ 
in two parts (see eq. (3.12)). To perform the splitting, we consider the expression (3.6) with $M_{F}=\mathcal{M}$ and we rewrite its right-hand side as follows:

$$
\begin{aligned}
D(n, \mathcal{M})= & \int_{M_{0}^{2}}^{\left(R p_{T}^{\gamma}\right)^{2}} \frac{d k^{2}}{k^{2}} K(n) e^{\int_{k^{2}}^{\left(R p_{T}^{\gamma}\right)^{2}} \frac{d k^{\prime 2}}{k^{\prime 2}} P(n)} \cdot e^{\int_{\left(R p_{T}^{\gamma}\right)^{2}}^{\mathcal{M}^{2}} \frac{d k^{\prime 2}}{k^{\prime 2}} P(n)} \\
& +\int_{\left(R p_{T}^{\gamma}\right)^{2}}^{\mathcal{M}^{2}} \frac{d k^{2}}{k^{2}} K(n) e^{\int_{k^{2}}^{\mathcal{M}^{2}} \frac{d k^{\prime 2}}{k^{\prime 2}} P(n)} \\
& +e^{\int_{\left(R p_{T}^{\gamma}\right)^{2}}^{\mathcal{M}^{2}} \frac{d k^{\prime 2}}{k^{\prime 2}} P(n)} \cdot e^{\int_{M_{0}^{2}}^{\left(R p_{T}^{\gamma}\right)^{2}} \frac{d k^{\prime 2}}{k^{\prime 2}} P(n)} D\left(n, M_{0}\right)
\end{aligned}
$$

In the LL approximation the splitting on the right-hand side of eq. (3.11) acquires a geometrical meaning. We denote this approximation by introducing superscripts " $(0)$ " and we rewrite eq. (3.11) as follows:

$$
D^{(0)}(n, \mathcal{M})=E^{(0)}\left(n, \mathcal{M}, R p_{T}^{\gamma}\right) D^{(0)}\left(n, R p_{T}^{\gamma}\right)+D^{(0)}\left(n, \mathcal{M}, R p_{T}^{\gamma}\right) .
$$

The first term on the right-hand side of eq. (3.12) corresponds to the configurations in which there are partons inside the cone of radius $R$. This term has two factors. The factor $D^{(0)}\left(n, R p_{T}^{\gamma}\right)$ is the fragmentation function at the factorization scale $R p_{T}^{\gamma}$ (see eq. (3.6)),

$$
\begin{array}{r}
D^{(0)}\left(n, R p_{T}^{\gamma}\right)=\int_{M_{0}^{2}}^{\left(R p_{T}^{\gamma}\right)^{2}} \frac{d k^{2}}{k^{2}} K^{(0)}(n) e^{\int_{k^{2}}^{\left(R p_{T}^{\gamma}\right)^{2}} \frac{d k^{\prime 2}}{k^{\prime 2}} \frac{\alpha_{s}\left(k^{\prime 2}\right)}{2 \pi} P^{(0)}(n)} \\
+e^{\int_{M_{0}^{2}}^{\left(R p_{T}^{\gamma}\right)^{2}} \frac{d k^{\prime 2}}{k^{\prime 2}} \frac{\alpha_{s}\left(k^{\prime 2}\right)}{2 \pi} P^{(0)}(n)} D^{(0)}\left(n, M_{0}\right)
\end{array}
$$

and it corresponds to the contribution of all emitted partons that are contained in the cone of radius $R$. The accompanying factor $E^{(0)}\left(n, \mathcal{M}, R p_{T}^{\gamma}\right)$ is the following exponential factor

$$
E^{(0)}\left(n, \mathcal{M}, R p_{T}^{\gamma}\right)=e^{\int_{\left(R p_{T}^{\gamma}\right)^{2}}^{\mathcal{M}^{2}} \frac{d k^{\prime 2}}{k^{\prime 2}} \frac{\alpha_{\mathcal{S}}\left(k^{\prime 2}\right)}{2 \pi} P^{(0)}(n)},
$$

and it sums the effect of the partons emitted outside the cone. The second term on the right-hand side of eq. (3.12) is

$$
D^{(0)}\left(n, \mathcal{M}, R p_{T}^{\gamma}\right)=\int_{\left(R p_{T}^{\gamma}\right)^{2}}^{\mathcal{M}^{2}} \frac{d k^{2}}{k^{2}} K^{(0)}(n) e^{\int_{k^{2}}^{\mathcal{M}^{2}} \frac{d k^{\prime 2}}{k^{\prime 2}} \frac{\alpha_{s}\left(k^{\prime 2}\right)}{2 \pi} P^{(0)}(n)},
$$

and it corresponds to the configurations with no partons inside the cone of radius $R$.

We then consider eq. (3.8) with $M_{F}=\mathcal{M}$. Since $\mathcal{M} \sim p_{T}^{\gamma}$, the higher-order contributions $\sigma_{F}^{(k)}$ and $\sigma_{D}^{(k)}$ (see eqs. (3.9) and (3.10)) to $\sigma_{F}(n, \mathcal{M})$ and $\sigma_{D}(n, \mathcal{M})$ cannot produce a LL dependence on $\ln R$ (roughly speaking, these higher-order contributions are due to parton radiation at very large angles with respect to the photon). Therefore, we can replace $\sigma_{F}(n, \mathcal{M})$ and $\sigma_{D}(n, \mathcal{M})$ with their Born level contribution and, inserting eq. (3.12) in eq. (3.8) (with $M_{F}=\mathcal{M}$ ), we finally obtain the expression of the cross section that explicitly shows the structure of all the LL terms produced by the introduction of the 
auxiliary cone of radius $R$. This final expression is

$$
\begin{aligned}
\sigma(n)= & \frac{\alpha_{s}}{\pi} \sigma_{D}^{\text {Born }}(n)+\left(\frac{\alpha_{s}}{\pi}\right)^{2} \sigma_{F}^{\text {Born }}(n) D^{(0)}\left(n, \mathcal{M}, R p_{T}^{\gamma}\right) \\
& +\left(\frac{\alpha_{s}}{\pi}\right)^{2} \sigma_{F}^{\text {Born }}(n) E^{(0)}\left(n, \mathcal{M}, R p_{T}^{\gamma}\right) D^{(0)}\left(n, R p_{T}^{\gamma}\right)+\ldots
\end{aligned}
$$

where the dots on the right-hand side denote contributions beyond the leading logarithmic approximation.

The first-order perturbative expansion of the expression (3.16) can directly be compared with the discussion of section 2. Expanding eqs. (3.13)-(3.15) up to the first order, we obtain

$$
\begin{aligned}
D^{(0)}\left(n, R p_{T}^{\gamma}\right)= & D^{(0)}\left(n, M_{0}\right)+K^{(0)}(n) \ln \left(\frac{\left(R p_{T}^{\gamma}\right)^{2}}{M_{0}^{2}}\right) \\
& +\frac{\alpha_{s}}{2 \pi} P^{(0)}(n) \ln \left(\frac{\left(R p_{T}^{\gamma}\right)^{2}}{M_{0}^{2}}\right) D^{(0)}\left(n, M_{0}\right)+\cdots, \\
E^{(0)}\left(n, \mathcal{M}, R p_{T}^{\gamma}\right)= & 1+\frac{\alpha_{s}}{2 \pi} P^{(0)}(n) \ln \left(\frac{\mathcal{M}^{2}}{\left(R p_{T}^{\gamma}\right)^{2}}\right)+\cdots, \\
D^{(0)}\left(n, \mathcal{M}, R p_{T}^{\gamma}\right)= & K^{(0)}(n) \ln \left(\frac{\mathcal{M}^{2}}{\left(R p_{T}^{\gamma}\right)^{2}}\right)+\cdots .
\end{aligned}
$$

Note that the contribution from single-parton radiation inside the cone depends logarithmically on the ratio $\left(R p_{T}^{\gamma}\right)^{2} / M_{0}^{2}$ (see eq. (3.17)), while the analogous contribution from ouside the cone depends logarithmically on the ratio $\mathcal{M}^{2} /\left(R p_{T}^{\gamma}\right)^{2}$ (see eqs. (3.18) and (3.19)). Note also that, in eqs. (3.17)-(3.19), the terms that are proportional to $K^{(0)}$ derive from the HO correction to the Born level direct cross section (see figure 1), while those that are proportional to $P^{(0)}$ derive from the HO correction to the Born level fragmentation cross section (see figure 2). Inserting eqs. (3.17)-(3.19) in the expression (3.16), we obtain the HO contributions to the Born level cross sections. The HO contribution to the Born level direct cross section is

$$
\left(\frac{\alpha_{s}}{\pi}\right)^{2} \sigma_{F}^{\text {Born }}(n)\left[K^{(0)}(n) \ln \left(\frac{\left(R p_{T}^{\gamma}\right)^{2}}{M_{0}^{2}}\right)+K^{(0)}(n) \ln \left(\frac{\mathcal{M}^{2}}{\left(R p_{T}^{\gamma}\right)^{2}}\right)\right]
$$

where the first term reproduces the result of eq. (2.1) (regularized by $M_{0}^{2}$ ) and the second term reproduces the logarithmic dependence on $R$ coming from the phase space part outside the cone (see section 2). Analogously, the HO contribution to the Born level fragmentation component (figure 2) is

$$
\left(\frac{\alpha_{s}}{\pi}\right)^{2} \sigma_{F}^{\text {Born }}(n) \frac{\alpha_{s}}{2 \pi}\left[P^{(0)}(n) \ln \left(\frac{\left(R p_{T}^{\gamma}\right)^{2}}{M_{0}^{2}}\right)+P^{(0)}(n) \ln \left(\frac{\mathcal{M}^{2}}{\left(R p_{T}^{\gamma}\right)^{2}}\right)\right] D^{(0)}\left(n, M_{0}\right)
$$

where the two terms reproduce the logarithmic contributions discussed in the final part of section 2 . 
Until now, we have explicitly considered the inclusive cross section and no isolation criterion has been imposed on the partonic (hadronic) transverse energy inside the smallsize cone. Therefore, the LL dependence on $R$ actually cancels on the right-hand side of eq. (3.16) (the addition of the last two terms in the right-hand side indeed reconstructs the fragmentation function $D^{(0)}(n, \mathcal{M})$, which is independent of $R$ order-by-order in the perturbative expansion). The discussion of the present section aimed at paving the way for the isolated case. Indeed, the resummation of the $\ln R$ dependence in the isolated cross section can straightforwardly be carried out on the basis of the decomposition in the right-hand side of eq. (3.16).

\section{Isolated cross section and resummation of the $\ln R$ dependence}

To study the isolated cross section we use a more refined notation. We explicitly reintroduce the parton indices, and we return (from Mellin space) to the configuration space, since the isolation constraint on transverse energies is directly applied to momentum fractions (see, e.g., the constraint on $z$ in eqs. (2.5) and (2.6)).

The isolated cross section $d \sigma^{i s} / d p_{T}^{\gamma} d \eta^{\gamma}$ with the cone isolation criterion of eqs. (1.1) and (1.2) is simply denoted by $\sigma^{i s}\left(p^{\gamma} ; z_{\text {cut }}, R\right)$, with (see eq. (2.5))

$$
z_{\text {cut }}=\frac{p_{T}^{\gamma}}{E_{T}^{\text {iso }}+p_{T}^{\gamma}} .
$$

The corresponding QCD factorization formula (analogous to eq. (3.8)) is written as in eq. (4.14) of ref. [38]:

$$
\begin{aligned}
\sigma^{i s}\left(p^{\gamma} ; z_{\text {cut }}, R\right)= & \sum_{a} \int_{0}^{1} \frac{d z}{z} \widehat{\sigma}^{a, i s}\left(\frac{p^{\gamma}}{z} ; \frac{z_{\text {cut }}}{z}, R ; \mu, M, M_{F}\right) D_{a}^{\gamma}\left(z ; M_{F}\right) \Theta\left(z-z_{\text {cut }}\right) \\
& +\widehat{\sigma}^{\gamma, i s}\left(p^{\gamma} ; z_{\text {cut }}, R ; \mu, M, M_{F}\right) .
\end{aligned}
$$

The subprocess cross sections $\widehat{\sigma}^{a, i s}$ and $\widehat{\sigma}^{\gamma, i s}$ are obtained by convolutions of partonic cross sections with the parton distribution functions of the two colliding hadrons. These convolutions are not explicitly denoted throughout the paper. The scale $M$ is the factorizaion scale of the parton distribution functions, and $\mu$ is the renormalization scale of the QCD coupling $\alpha_{s}$.

The QCD perturbative expansion of the partonic cross sections leads to a corresponding expansion of the subprocess cross sections. We write the expansion as follows (see eqs. (4.18) and (4.19) in ref. [38]):

$$
\begin{aligned}
\widehat{\sigma}^{\gamma, i s}\left(p ; z_{c}, R ; \mu, M, M_{F}\right)= & \left(\frac{\alpha_{s}(\mu)}{\pi}\right) \sigma_{\gamma}^{\text {Born }}(p ; M) \\
& +\left(\frac{\alpha_{s}(\mu)}{\pi}\right)^{2} \sigma_{H O}^{\gamma, i s}\left(p ; z_{c}, R ; \mu, M, M_{F}\right)+\mathcal{O}\left(\alpha_{s}^{3}\right), \\
\widehat{\sigma}^{a, i s}\left(p ; z_{c}, R ; \mu, M, M_{F}\right)= & \left(\frac{\alpha_{s}(\mu)}{\pi}\right)^{2} \sigma_{a}^{\text {Born }}(p ; M) \\
& +\left(\frac{\alpha_{s}(\mu)}{\pi}\right)^{3} \sigma_{H O}^{a, i s}\left(p ; z_{c}, R ; \mu, M, M_{F}\right)+\mathcal{O}\left(\alpha_{s}^{4}\right) .
\end{aligned}
$$


The evaluation of the isolated cross section $\sigma^{i s}$ up to the NLO requires the computation of the first two terms on the right-hand side of eqs. (4.3) and (4.4). This NLO computation, with the exact dependence on $R$ (i.e., without any small- $R$ approximations) is performed in ref. [38] and it is implemented in the programme Jetphox.

As discussed in the previous sections, at small values of $R$, the $\mathrm{HO}$ terms $\sigma_{H O}^{\gamma, i s}$ and $\sigma_{H O}^{a, i s}$ in eqs. (4.3) and (4.4) contain a contribution that is proportional to $\ln R$. Additional powers of $\ln R$ appear at still higher orders in the $\alpha_{s}$ expansion. The direct component $\widehat{\sigma}^{\gamma, i s}$ in eq. (4.3) contains logarithmic terms of the type $\alpha_{s}^{m+1}\left(\alpha_{s} \ln R\right)^{k}$, and the fragmentation component $\widehat{\sigma}^{a, i s}$ in eq. (4.4) contains logarithmic terms of the type $\alpha_{s}^{m+2}\left(\alpha_{s} \ln R\right)^{k}$. The LL terms are those with $m=0$ (and $k=1,2,3, \ldots$ ).

The resummation of the LL terms (the subscript notation [ ] LL $_{\text {denotes the LL }}$ accuracy) leads to the following result

$$
\begin{aligned}
& {\left[\sigma^{i s}\left(p^{\gamma} ; z_{\text {cut }}, R\right)\right]_{\mathrm{LL}} } \\
&=\frac{\alpha_{s}(\mu)}{\pi} \sigma_{\gamma}^{\text {Born }}\left(p^{\gamma} ; M\right)+\left(\frac{\alpha_{s}(\mu)}{\pi}\right)^{2} \sum_{a} \int_{0}^{1} \frac{d z}{z} \sigma_{a}^{\text {Born }}\left(\frac{p^{\gamma}}{z} ; M\right) D_{a}^{(0)}\left(z ; \mathcal{M}, R p_{T}^{\gamma}\right) \\
&+\left(\frac{\alpha_{s}(\mu)}{\pi}\right)^{2} \sum_{a, b} \int_{0}^{1} \frac{d z}{z} \sigma_{a}^{\text {Born }}\left(\frac{p^{\gamma}}{z} ; M\right) \times \\
& \times \int_{z}^{1} \frac{d x}{x} E_{a b}^{(0)}\left(\frac{z}{x} ; \mathcal{M}, R p_{T}^{\gamma}\right) D_{b}^{\gamma(0)}\left(x ; R p_{T}^{\gamma}\right) \Theta\left(x-z_{\text {cut }}\right)
\end{aligned}
$$

where $\sigma_{\gamma}^{\text {Born }}$ and $\sigma_{a}^{\text {Born }}$ are the Born level subprocess cross sections in eqs. (4.3) and (4.4), and $E_{a b}^{(0)}$ and $D_{a}^{(0)}$ are the customary QCD evolution operators at the LL order. The expression of the parton evolution operator $E_{a b}^{(0)}\left(z ; \mathcal{M}, R p_{T}^{\gamma}\right)$ is obtained from eq. (3.14) by reinserting the explicit dependence on the parton indices; the $n$-moments with respect to the momentum fraction $z$ are given by the exponentiated formula (3.14) by replacing the flavour NS kernel $P^{(0)}(n)$ with the DGLAP matrix kernel $P_{a b}^{(0)}(n)$. A corresponding replacement is applied to eq. (3.15) to obtain the photon evolution operator $D_{a}^{(0)}\left(z ; \mathcal{M}, R p_{T}^{\gamma}\right)$; the explicit expression of this operator is

$$
D_{a}^{(0)}\left(z ; \mathcal{M}, R p_{T}^{\gamma}\right)=\sum_{b} \int_{\left(R p_{T}^{\gamma}\right)^{2}}^{\mathcal{M}^{2}} \frac{d k^{2}}{k^{2}} \int_{z}^{1} \frac{d x}{x} E_{a b}^{(0)}\left(\frac{z}{x} ; \mathcal{M}, k\right) K_{b}^{(0)}(x) .
$$

The LL resummation formula (4.5) is directly derived by supplementing the right-hand side of eq. (3.16) with the isolation constraint on the partonic transverse energy. Each of the three terms in the right-hand side of eq. (4.5) is in direct correspondence with the analogous term in eq. (3.16). The first term in the right-hand side of eq. (3.16) is the Born level direct contribution, and the second term corresponds to kinematical configurations with no partons inside the isolation cone. Since in these two cases the photon is evidently isolated, these terms give the same contribution to the inclusive cross section of eq. (3.16) and to the isolated cross section of eq. (4.5). In the third term on the right-hand side of eq. (3.16), the operator $E^{(0)}$ embodies parton radiation outside the isolation cone, while the photon fragmentation function $D^{(0)}\left(n, R p_{T}^{\gamma}\right)$ embodies parton radiation inside the cone. 
Therefore, only the fragmentation function must be isolated by applying the transverseenergy isolation constraint of eq. (1.2). This isolation constraint leads to the momentum fraction cut $x>z_{\text {cut }}$ that is explicitly implemented in eq. (4.5).

In the LL resummed expression (4.5), the fragmentation function $D_{b}^{\gamma(0)}$ is evaluated at the evolution scale $R p_{T}^{\gamma}$. Therefore, it is interesting to make a comparison of eq. (4.5) with the NLO cross section by choosing $M_{F}=R p_{T}^{\gamma}$ in the NLO expression. Setting $M_{F}=R p_{T}^{\gamma}$ in eqs. (4.2)-(4.4), the NLO result effectively resums the $\ln R$ terms produced by parton radiation inside the isolation cone. However, the corresponding $\mathrm{HO}$ subprocess cross sections $\sigma_{H O}^{\gamma, i s}\left(M_{F}=R p_{T}^{\gamma}\right)$ and $\sigma_{H O}^{a, i s}\left(M_{F}=R p_{T}^{\gamma}\right)$ of eqs. (4.3) and (4.4) still contain a residual $\ln R$ term (which is due to partons radiated outside the isolation cone) that is not resummed by the NLO result. In the resummation formula (4.5), this residual $\ln R$ term at NLO is produced by the first-order expansion of $D_{a}^{(0)}\left(z ; \mathcal{M}, R p_{T}^{\gamma}\right)$ (which contributes to $\left.\sigma_{H O}^{\gamma, i s}\left(M_{F}=R p_{T}^{\gamma}\right)\right)$ and $E_{a b}^{(0)}\left(z / x ; \mathcal{M}, R p_{T}^{\gamma}\right)$ (which contributes to $\left.\sigma_{H O}^{a, i s}\left(M_{F}=R p_{T}^{\gamma}\right)\right)$.

Using eq. (4.6) and the exponentiated form (see eq. (3.14)) of the evolution operator $E^{(0)}\left(\mathcal{M}, R p_{T}^{\gamma}\right)$, the expression (4.5) can be used to explicitly resums the LL contributions to the isolated cross section at small values of $R$. Note, however, that the scale $R p_{T}^{\gamma}$ must be sufficiently larger than the typical scale of the non-perturbative domain (e.g., $R p_{T}^{\gamma} \geq M_{0}$ where $M_{0}$ is the cut-off scale in eq. (3.6)). At very small values of $R$ (and $R p_{T}^{\gamma}$ ), the photon fragmentation function $D_{b}^{\gamma}\left(x ; R p_{T}^{\gamma}\right)$ in eq. (4.5) and, more generally, the isolated cross section become sensitive to sizeable non-perturbative effects that are not taken into account by the perturbative QCD factorization formula (4.2).

The resummation of the $\ln R$ terms can be generalized beyond the LL level of eq. (4.5). We have worked out the formal generalization to arbitrary logarithmic accuracy. The allorder generalization and the explicit treatment of next-to-leading logarithmic (NLL) terms will be presented in a forthcoming paper. The extension of resummation to other isolation criteria (e.g., the criterion in section 6) is in progress. In the next section, we explicitly apply the LL resummation formula of eq. (4.5), and we present ensuing quantitative results for Tevatron and LHC kinematical configurations.

\section{Quantitative results}

We have implemented the LL resummation of the $\ln R$ terms in the programme Jetphox. This implementation supplements the complete NLO result [38] (which has the exact dependence on $R$ ) with the resummation of all the LL terms beyond the NLO. The complete NLO result is added to a 'subtracted version' of the LL resummation formula (4.5). This subtracted version avoids double counting of perturbative terms. It is obtained by considering the LL formula (4.5) and by explicitly subtracting from it the terms that are obtained by expanding the same formula up to the NLO.

We add some comments about our actual implementation of resummation in Jetphox. We want results with consistent NLO accuracy (and exact dependence on $R$ ) for both the direct and fragmentation contributions and, therefore, we have to use NLO fragmentation functions (and parton distribution functions). To this purpose, in the LL resummation formula (4.5), the LO fragmentation function $D^{(0)}$ can be replaced by the NLO one: this 
replacement is permitted, since it produces corrections beyond the LL approximation. Then we note that, due to the isolation cut $x>z_{\text {cut }}$, the contribution of the fragmentation function $D_{b}^{\gamma}\left(x ; R p_{T}^{\gamma}\right)$ is small, so that the impact of the resummed factor $E^{(0)}\left(\mathcal{M}, R p_{T}^{\gamma}\right.$, ) in the third term on the right-hand side of eq. (4.5) is not significant. For the sake of numerical simplicity, this resummed factor is replaced by its truncation at $\mathcal{O}\left(\alpha_{s}\right)$ :

$$
E^{(0)}\left(z / x ; \mathcal{M}, R p_{T}^{\gamma}\right) \rightarrow \delta(1-z / x)+\frac{\alpha_{s}}{2 \pi} P^{(0)}(z / x) \ln \left(\frac{\mathcal{M}^{2}}{\left(R p_{T}^{\gamma}\right)^{2}}\right) .
$$

We observe that the replacement in eq. (5.1) implies that the third term on the righthand side of eq. (4.5) does not produce any LL terms beyond the NLO, provided the NLO perturbative expansion is carried out at the factorization scale $M_{F}=R p_{T}^{\gamma}$ (this observation is equivalent to that made in the final part of section 4 , where we pointed out that the NLO result with $M_{F}=R p_{T}^{\gamma}$ effectively resums the LL terms produced by parton radiation inside the isolation cone). Therefore, we can consider the NLO result of Jetphox with $M_{F}=R p_{T}^{\gamma}$ and supplement it with the LL terms produced by the sole direct component of the cross section in eq. (4.5). In practical terms, the resummation part to be added to the NLO cross section $\sigma^{i s}\left(p^{\gamma} ; z_{\text {cut }}, R\right)$ of eqs. (4.2)-(4.4) is the following contribution:

$$
+\left(\frac{\alpha_{s}(\mu)}{\pi}\right)^{2} \sum_{a} \int_{0}^{1} \frac{d z}{z} \sigma_{a}^{\text {Born }}\left(\frac{p^{\gamma}}{z} ; M\right)\left\{D_{a}^{(0)}\left(z ; \mathcal{M}, R p_{T}^{\gamma}\right)-K_{a}^{(0)}(z) \ln \frac{\mathcal{M}^{2}}{\left(R p_{T}^{\gamma}\right)^{2}}\right\}
$$

This contribution corresponds to the first two terms in the right-hand side of eq. (4.5), after subtraction of their NLO expansion which is already contained in the $\mathcal{O}\left(\alpha_{s}^{2}(\mu)\right)$ term of the expression (4.5) (the second term in the curly bracket of eq. (5.2) is exactly the first-order expansion of $D_{a}^{(0)}\left(z ; \mathcal{M}, R p_{T}^{\gamma}\right)$, which is the first term in the curly bracket).

In this section we compare the outputs of Jetphox obtained without and with resummation.

We start our presentation with kinematics relevant to Tevatron experiments that we already studied in ref. [38]. We consider proton-antiproton collisions at the centre-of-mass energy $\sqrt{s}=1.8 \mathrm{TeV}$, and we use $p_{T}^{\gamma}=15 \mathrm{GeV},-0.9 \leq \eta^{\gamma} \leq 0.9$ and $\varepsilon=E_{T}^{\text {iso }} / p_{T}^{\gamma}=0.1333$ (i.e., $z_{\text {cut }}=0.88235$ and $E_{T}^{\text {iso }}=2 \mathrm{GeV}$ ). The calculations are done with $N_{f}=5$ flavours of massless quarks. The renormalization scale $(\mu)$ and the factorization scale $(M)$ of the parton distribution functions are set to be equal to $p_{T}^{\gamma} / 2$. As for the factorization scale of the photon fragmentation function, we study the cases with $M_{F}=p_{T}^{\gamma} / 2$ (conventional scale) and $M_{F}=R p_{T}^{\gamma}$ ("cone scale"). We use the CTEQ6M parton distribution functions [59]. The fragmentation functions are those of the BFG set II [57]. As we have already discussed, we use NLO fragmentation functions and parton distribution functions. The resummed contribution of eq. (5.2) is calculated with $\mathcal{M}=p_{T}^{\gamma}$.

Our results are summarized in tables 1 and 2. Table 1 gives the details of the direct and fragmentation contributions to the isolated cross sections calculated with the "conventional" and "cone" scales, with or without resummation. We also give the results of the non-isolated cross sections. In table 2 one can find the behavior with $R$ of the total cross sections. The numerical values that we obtain at the Born level and at the NLO are not 


\begin{tabular}{|c|c|c|c|c|c|c|c|c|}
\hline \multicolumn{5}{|c|}{ DIRECT } & \multicolumn{4}{|c|}{ FRAGMENTATION } \\
\hline$R$ & Born & $\begin{array}{l}\mathrm{NLO} \\
p_{T}^{\gamma} / 2\end{array}$ & $\begin{array}{l}\text { NLO } \\
R p_{T}^{\gamma}\end{array}$ & $\begin{array}{c}\mathrm{NLO} \\
R p_{T}^{\gamma} \\
\text {-resummed }\end{array}$ & $\begin{array}{l}\text { Born } \\
p_{T}^{\gamma} / 2\end{array}$ & $\begin{array}{l}\text { Born } \\
R p_{T}^{\gamma}\end{array}$ & $\begin{array}{l}\text { NLO } \\
p_{T}^{\gamma} / 2\end{array}$ & $\begin{array}{l}\text { NLO } \\
R p_{T}^{\gamma}\end{array}$ \\
\hline .9 & 1972 & 3737 & 3586 & 3592 & 291 & 348 & 516 & 700 \\
\hline .7 & 1972 & 3951 & 3864 & 3851 & 291 & 324 & 555 & 662 \\
\hline .5 & 1972 & 4197 & 4197 & 4171 & 291 & 291 & 597 & 597 \\
\hline .3 & 1972 & 4532 & 4663 & 4593 & 291 & 245 & 654 & 496 \\
\hline .1 & 1972 & 5203 & 5616 & 5294 & 291 & 165 & 764 & 318 \\
\hline No isol & 1972 & 3655 & & & 1875 & & 2044 & \\
\hline
\end{tabular}

Table 1. Variation with $R$ of the various contributions to the cross sections (the values are expressed in $\mathrm{pb} / \mathrm{GeV}$ ) at $\sqrt{s}=1.8 \mathrm{TeV}$.

\begin{tabular}{|c|c|c|c|}
\hline$R$ & $\begin{array}{c}\text { NLO } \\
p_{T}^{\gamma} / 2\end{array}$ & $\begin{array}{c}\text { NLO } \\
R p_{T}^{\gamma}\end{array}$ & $\begin{array}{c}\text { NLO } \\
R p_{T}^{\gamma} \\
\text {-resummed }\end{array}$ \\
\hline .9 & 4253 & 4286 & 4292 \\
.7 & 4506 & 4526 & 4513 \\
.5 & 4794 & 4794 & 4768 \\
.3 & 5186 & 5159 & 5089 \\
.1 & 5967 & 5934 & 5612 \\
\hline No isol & 5699 & & \\
\hline
\end{tabular}

Table 2. Variation with $R$ of the total cross sections (in $\mathrm{pb} / \mathrm{GeV}$ ) at $\sqrt{s}=1.8 \mathrm{TeV}$.

identical to those of ref. [38], since a different set (the MRST-99 set) of parton distribution functions was used in ref. [38].

The first point to note in table 1 is the strong effect of isolation on the fragmentation component. Another noticeable point is the increase of the NLO direct contribution when $R$ decreases; this increase is in agreement with the $\ln R$ enhancement shown in the expression (2.6). In particular, the isolated contribution can be larger than the nonisolated contribution (we report the non-isolated reference values at the factorization scale $\left.M_{F}=p_{T}^{\gamma} / 2\right)$. No resummation is involved in the calculation of the non-isolated crosssection. The resummed direct cross section is smaller (by about $5 \%$ at $R=0.1$ ) than the cross section without resummation. The fragmentation component is quite sensitive to $R$ already at the Born level through the $M_{F}$ dependence on $R$ (this is because the typical behaviour of the fragmentation function is $\left.D\left(R p_{T}^{\gamma}\right) \sim \ln \left(R p_{T}^{\gamma} / M_{0}\right)\right)$.

The NLO cross sections are given in table 2. In the first column, at the value $R=0.1$ we notice a violation of unitarity since the non-isolated cross section at the reference scale $M_{F}=p_{T}^{\gamma} / 2$ is smaller than the isolated one for both the scale choices $M_{F}=p_{T}^{\gamma} / 2$ and $M_{F}=R p_{T}^{\gamma}$. When $R$ becomes small, the $\ln R$ terms has to be resummed: this is performed in the rightmost column of table 2 , and we notice that resummation does restore unitarity. 


\begin{tabular}{|c|c|c|c|}
\hline$R$ & $\begin{array}{c}\text { NLO } \\
p_{T}^{\gamma} / 2\end{array}$ & $\begin{array}{c}\text { NLO } \\
R p_{T}^{\gamma}\end{array}$ & $\begin{array}{c}\text { NLO } \\
R p_{T}^{\gamma} \\
\text {-resummed }\end{array}$ \\
\hline .5 & 3.59 & 3.59 & 3.57 \\
.3 & 3.86 & 3.85 & 3.81 \\
.1 & 4.35 & 4.34 & 4.19 \\
.06 & 4.56 & 4.55 & 4.24 \\
\hline No isol & 4.29 & & \\
\hline
\end{tabular}

Table 3. Variation with $R$ of the total cross sections (in $\mathrm{pb} / \mathrm{GeV}$ ) at $\sqrt{s}=7 \mathrm{TeV}$.

Notwithstanding we also notice that at $R=0.1$ the resummed cross section is about $5 \%$ smaller than the NLO cross section. Therefore the effect of resummation is not very large at $R=0.1$, although it is qualitatively and conceptually important regarding the restoration of unitarty. We cannot explore lower values of $R$ because the scale at which the fragmentation function is calculated becomes too small.

To study smaller values of $R$ we turn to the LHC kinematics and we consider higher values of $p_{T}^{\gamma}$. We consider proton-proton collisions at $\sqrt{s}=7 \mathrm{TeV}, p_{T}^{\gamma}=100 \mathrm{GeV}$ and $\left|\eta^{\gamma}\right|<0.6$. The energy isolation parameter is $\varepsilon=0.04$ (i.e., $z_{\text {cut }}=0.9615$ and $E_{T}^{\text {iso }}=4 \mathrm{GeV}$ ) and the radius of the isolation cone is varied in the range $0.06 \leq R \leq 0.5$. The parton distributions functions, the fragmentation functions and the scale choices are the same as in the Tevatron study reported in tables 1 and 2. Our LHC results are summarized in table 3 .

The first two columns of table 3 show that the NLO cross section is very stable with respect to changes of the factorization scale $M_{F}$ when the photon is isolated. Here we also note a violation of unitarity for small values of $R(R \leq 0.1)$. The rightmost column displays the effect of resummation. It is small, and it is of the order of $7 \%$ at $R=0.06$. Unitarity is no more violated down to the very small value $R=0.06$. This very small value of $R$ is however extreme; the experimental isolation cones typically have a radius bigger than 0.3 for which the effect of resummation is even smaller $(\leq 1 \%)$.

The issue of the violation of unitarity in the NLO calculation of the isolated cross section with narrow cones is quantified, throughout this section, by the numerical comparison with the non-isolated photon cross section at the reference fragmentation scale $M_{F}=p_{T}^{\gamma} / 2$. Obviously the choice of reference scale has some degree of arbitrariness, and any other commonly used choice, such as $M_{F}=p_{T}^{\gamma}$ or $2 p_{T}^{\gamma}$ would affect the numerical results somewhat (we have explicitly checked that the use of these scales does not significantly change the values of the non-isolated cross section). However the main trends of the results presented in this section are evident, and are summarized as follows. 1) The violation of unitarity observed in NLO cross sections for photons isolated with narrow cones (with aperture $R \ll 1$ ) is due to the inappropriate truncation, to fixed order, of an expansion that involves terms logarithmically enhanced in $\ln R$. The violation cannot be cured by simply adjusting the scales. Instead the resummation of these logarithmically enhanced terms, as we have performed, cures this unitarity puzzle down to values of $R$ that 
are much lower than the ones physically used in colliders experiments. 2) Notwithstanding, down to at least $R=0.3$, which is already smaller than the value 0.4 commonly used in colliders experiments, this resummation amounts to a small, few $\%$ correction with respect to the NLO calculation using a standard choice of $M_{F}$, and this correction is smaller than the usual scale uncertainty of the NLO prediction. The unitarity puzzle is therefore not an issue in NLO predictions with the cones sizes that are commonly used in colliders experiments. Moreover, resummation can be regarded and used as a complementary and additional theoretical tool that is available to asses the reliability of the QCD predictions and to quantify their uncertainties in studies with moderately-small values of $R$.

\section{Implications for a hollow-cone criterion}

Using the standard cone criterion or an alternative one, such as the Frixione criterion [35] or one of its discretized versions [36, 37], it may be experimentaly difficult to apply a cut on the accompanying energy in the region where the electromagnetic shower develops in the detector, since it may be difficult to disentangle the accompanying energy from the photon energy inside that region. The shape and size of this region are detector dependent. To first approximation, the electromagnetic shower roughly fills a cone of radius $r \sim 0.1$ : considering the standard cone criterion, this would correspond to an inner narrow cone of inefficient isolation inside the usual cone of radius $R$. For a 'hollow' cone with an inner (empty) cone of such a small size $(r \sim 0.1)$, the issue of the resummation of $\ln r$ contributions can matter. In this section, we perform a first explorative investigation of this issue.

We consider a hollow-cone variant of the standard cone isolation criterion in eqs. (1.1) and (1.2). A cone of radius $R$ around the photon direction is still considered, but the upper limit $E_{T}^{\text {iso }}$ is enforced on the hadronic transverse energy inside an annular region $\mathcal{C}_{\gamma}^{\text {holl. }}(R, r)$ of width $R-r$, rather than on the hadronic transverse energy inside the whole cone. The constraint in eq. (1.2) is thus replaced by

$$
\sum_{k \in \mathcal{C}_{\gamma}^{\text {holl. }}(R, r)} E_{T}^{(k)}<E_{T}^{\text {iso }} .
$$

Therefore, no isolation is applied inside the innermost region delimited by the small cone of radius $r$ (thus coined 'hollow', in the sense of free of any isolation constraint, in what follows). The usual isolation is instead implemented outside the innermost cone of radius $r$.

Note that the hollow-cone criterion selects photons that are less isolated than those selected by the standard cone criterion. Therefore, at fixed values of $E_{T}^{\text {iso }}$ for both criteria, the corresponding cross sections fulfil the physical requirement

$$
\sigma\left(p^{\gamma}\right) \geq \sigma^{\text {holl }}\left(p^{\gamma} ; R, r\right) \geq \sigma^{i s}\left(p^{\gamma} ; R\right)
$$

where $\sigma\left(p^{\gamma}\right)$ is the inclusive cross section with no isolation, $\sigma^{i s}\left(p^{\gamma} ; R\right)$ is the cone isolation cross section (i.e., the cross section considered in sects. 4 and 5$))$ and $\sigma^{\text {holl }}\left(p^{\gamma} ; R, r\right)$ is the cross section for the hollow-cone criterion. 


\begin{tabular}{|c|cc|cc|c|}
\hline \multirow{2}{*}{$\begin{array}{c}\text { cone } \\
\text { type }\end{array}$} & \multicolumn{2}{|c|}{ direct } & \multicolumn{2}{|c|}{ fragmentation } & total \\
\cline { 2 - 6 } & Born & NLO & Born & NLO & NLO \\
\hline standard & 2.08 & 3.56 & .077 & .165 & 3.73 \\
hollow & 2.08 & 3.09 & .91 & .43 & 3.52 \\
\hline
\end{tabular}

Table 4. NLO cross sections (in pb/GeV) at $\sqrt{s}=7 \mathrm{TeV}$, with $\varepsilon=0.04$ and $M_{F}=r p_{T}^{\gamma}$. Comparison of the isolated cross sections for the standard cone $(R=0.4)$ vs. the hollow-cone $(R=0.4, r=0.1)$ criteria.

The factorization structure of the perturbative QCD calculation of the hollow-cone cross section is discussed in the final part of section 4 of ref. [38]. Here we briefly comment on the expected behaviour of the NLO cross section in the case of a very small innermost cone $(r \rightarrow 0)$. As discussed in section 2 , the presence of the boundary of a cone of small radius $R_{0}$ (we use a generic $R_{0}$ to allow us to make a following distinction between $R$ and $r$ ) around the photon direction produces $\ln R_{0}$-terms in the NLO calculation. Typically, single-parton radiation inside the cone leads to negative $\ln R_{0}$-terms, while parton radiation just outside the cone leads to analogous positive terms. Within the standard cone criterion $\left(R_{0}=R\right.$ ), energy isolation is applied inside the cone thus suppressing the negative $\ln R$ terms, and the NLO cross section receives a total positive contribution from the $\ln R$-terms. In the hollow-cone case $\left(R_{0}=r\right)$, the situation is reversed with respect to the standard cone criterion: energy isolation is now imposed outside the cone $r$ instead of inside it. This suppresses the positive $\ln r$-terms, and the NLO cross section $\sigma^{\text {holl }}\left(p^{\gamma} ; R, r\right)$ receives a total negative contribution from the $\ln r$-terms. Eventually, in the limit $r \rightarrow 0$ (at fixed $R$ ), we can expect a violation of unitarity of the NLO result since $\sigma^{\text {holl }}\left(p^{\gamma} ; R, r\right)$ can become smaller than $\sigma^{i s}\left(p^{\gamma} ; R\right)$, thus violating the lower bound of the physical requirement in eq. (6.2).

We use Jetphox to compute the NLO cross section for the hollow-cone criterion, and we present quantitative results by considering the LHC kinematical configuration already discussed in section 5. We consider a hollow-cone criterion with the inner cone of radius $r=0.1$ and the outer cone of radius $R=0.4$. The results for the NLO cross sections are given in table 4 , where they are also compared with the corresponding standard-cone cross section (with $R=0.4$ ).

The NLO hollow-cone cross section in table 4 is computed by using the fragmentationscale choice $M_{F}=r p_{T}^{\gamma}$. This choice is motivated by analogy with the discussion in the previous sections: setting $M_{F}=r p_{T}^{\gamma}$, we effectively resum part of the higher-order $\ln r$ terms (the part from parton radiation inside the inner cone). For the sake of direct comparison, the NLO standard-cone cross section reported in table 4 is computed by using the same numerical value, $M_{F}=0.1 p_{T}^{\gamma}$, that is used in the hollow-cone cross section. The results of the standard-cone cross section that are obtained with different choices of $M_{F}$ $\left(M_{F}=p_{T}^{\gamma} / 2, M_{F}=R p_{T}^{\gamma}\right)$ at the NLO and with LL resummation were reported in table 3. All these values of the standard-cone cross section at $R=0.4$ (the value in table 4 , and the values that can be inferred from table 3 ) are numerically very similar. Therefore, in the context of the discussion in this section, we can state that we have a reliable estimate of the standard-cone cross section at $R=0.4$. 
The results in table 4 show that the total (i.e. direct + fragmentation) NLO predictions for the standard cone criterion and the hollow-cone criterion do not differ much, despite the drastic absence of isolation in the inner cone. The two NLO results depart from each other by about $6 \%$, and they would differ even less if some loose isolation were implemented inside the inner cone. Indeed, our hollow-cone criterion with no isolation inside the inner cone is an extreme simplification for modelling the experimental region of inefficient isolation (for instance, CMS analyses impose a veto on charged tracks in the close vicinity of the photon candidates).

Nonetheless, the quantitative results in table 4 also show that the hollow-cone cross section is smaller than the standard cone cross section, thus violating the bound $\sigma^{\text {holl }}\left(p^{\gamma} ; R, r\right) \geq \sigma^{i s}\left(p^{\gamma} ; R\right)$ that is set by the physical requirement in eq. (6.2). This violation is an artifact of the NLO truncation of the perturbative QCD calculation. The misbehaviour of the NLO cross section for the hollow-cone criterion is due to the presence of large and negative $\ln r$ terms, as discussed at the beginning of this section. We have quantitatively checked that this misbehaviour of the NLO calculation persists and it is enhanced by decreasing further the values of $r$. In particular, at very small values of $r$, the NLO corrections are found to be very large especially for the fragmentation component of the cross section, which eventually becomes (increasingly) negative. The different relative importance of the fragmentation component compared with the direct component comes from the absence of isolation inside the inner cone. Without the resummation of $\ln r$ terms, we cannot expect a sound theoretical result for the hollow-cone criterion at very small values of $r$. We postpone the study of resummation of $\ln r$ terms for the hollow-cone criterion to future work, which is in progress. In particular, in the hollow-cone case, very small values of $r$ and, consequently, very large values of $\ln (1 / r)$ are considered. A reliable quantitative treatment of resummation effects may thus requires the LL contributions and the inclusion of terms at the NLL level, which may have a not negligible quantitative role.

\section{Conclusions}

We have considered the standard cone isolation criterion that is used to measure promptphoton cross sections at $\mathrm{TeV}$ colliders. We have performed a detailed study of the dependence of the QCD theoretical predictions on size $R$ of the isolation cone. The dependence arises from the mismatch of parton radiation in the region inside the cone, which is submitted to isolation, vs. the region outside the cone, where no isolation is imposed. The dependence on the cone size $R$ is dominantly logarithmic at small $R$, and the NLO predictions become unreliable when $R$ becomes too small. We restored the reliability of the theoretical estimates by performing the resummation of this logarithmic dependence to LL accuracy in $R$. The resummation eventually amounts to the following procedure: the fragmentation scale is set to the value $M_{F} \sim R p_{T}^{\gamma}$ (as simply suggested from physical insight) to take into account the $\ln R$ dependence from parton radiation inside the cone, and an additional explicit resummation is performed to control the $\ln R$ dependence that arises from the region outside the cone, which is not submitted to isolation. We have implemented this resummation in the partonic Monte Carlo programme Jetphox, which also 
includes the non logarithmic $R$ dependence that is not negligible at the moderate value $R \sim 0.4$ that is used experimentally at the LHC. We have presented ensuing quantitative results that show how the resummation cures the instabilities of the NLO calculation down to very small values of $R$. At the typical values of $R(R \gtrsim 0.3)$ that are currently used in collider experiments, the resummation effects are small, and they are not larger than the size of the usual theoretical uncertainties of the NLO predictions.

We also explored the case of "hollow cone" isolation. The isolation is imposed in an annulus between two cones of radii $r$ and $R$, with $r<R$, whereas the regions outside the annulus, including the one inside the inner cone, is free from any isolation constraints. The cone with small radius $r$ aims at simulating the size of the electromagnetic shower that develops in the calorimeter. Considering the values $r=0.1$ and $R=0.4$, the corresponding NLO cross section does not differ much from the cross section with the standard cone isolation, and the difference would be even smaller by using some loose isolation inside the inner cone, rather than none as we did. However, we also noticed that the strict implementation of the hollow-cone criterion (with the extreme situation of absolutely no isolation inside the inner cone of radius $r$ ) can produce NLO inconsistencies, leading to quantitative values of the hollow-cone cross section that are smaller than those of the more-isolated cross section of the standard cone isolation. A proper resummation of $\ln r$ terms would be necessary to cure these NLO inconsistencies and to safely use the hollowcone criterion for perturbative QCD calculations at small values of $r$.

\section{Acknowledgments}

This work is supported in part by the Research Executive Agency (REA) of the European Union under the Grant Agreement number PITN-GA-2010-264564 (LHCPhenoNet).

Open Access. This article is distributed under the terms of the Creative Commons Attribution License which permits any use, distribution and reproduction in any medium, provided the original author(s) and source are credited.

\section{References}

[1] CDF collaboration, T. Aaltonen et al., Measurement of the inclusive isolated prompt

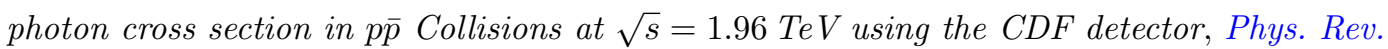
D 80 (2009) 111106 [arXiv:0910.3623] [INSPIRE].

[2] CDF collaboration, F. Abe et al., Properties of photon plus two-jet events in $\bar{p} p$ collisions at $\sqrt{s}=1.8 \mathrm{TeV}$, Phys. Rev. D 57 (1998) 67 [INSPIRE].

[3] CDF collaboration, F. Abe et al., Jet pseudorapidity distribution in direct photon events in $p \bar{p}$ collisions at $\sqrt{s}=1.8$ TeV, Phys. Rev. D 57 (1998) 1359 [INSPIRE].

[4] D0 collaboration, V. Abazov et al., Measurement of the isolated photon cross section in $p \bar{p}$ collisions at $\sqrt{s}=1.96 \mathrm{TeV}$, Phys. Lett. B 639 (2006) 151 [Erratum ibid. B 658 (2008) 285-289] [hep-ex/0511054] [INSPIRE].

[5] D0 collaboration, V. Abazov et al., Measurement of the differential cross-section for the production of an isolated photon with associated jet in p p collisions at $\sqrt{s}=1.96 \mathrm{TeV}$, Phys. Lett. B 666 (2008) 435 [arXiv: 0804.1107] [InSPIRE]. 
[6] D0 collaboration, V.M. Abazov et al., Azimuthal decorrelations and multiple parton interactions in photon+2 jet and photon+3 jet events in p $\bar{p}$ collisions at $\sqrt{s}=1.96 \mathrm{TeV}$, Phys. Rev. D 83 (2011) 052008 [arXiv:1101.1509] [INSPIRE].

[7] PHENIX collaboration, A. Adare et al., Direct-photon production in $p+p$ collisions at $\sqrt{s}=200$ GeV at midrapidity, Phys. Rev. D 86 (2012) 072008 [arXiv:1205.5533] [INSPIRE].

[8] ATLAS collaboration, Measurement of the inclusive isolated prompt photon cross-section in pp collisions at $\sqrt{s}=7 \mathrm{TeV}$ using $35 p b^{-1}$ of ATLAS data, Phys. Lett. B 706 (2011) 150 [arXiv: 1108.0253] [INSPIRE].

[9] ATLAS collaboration, Measurement of the production cross section of an isolated photon associated with jets in proton-proton collisions at $\sqrt{s}=7 \mathrm{TeV}$ with the ATLAS detector, Phys. Rev. D 85 (2012) 092014 [arXiv: 1203.3161] [InSPIRE].

[10] ATLAS collaboration, Measurement of high $p_{T}$ isolated prompt photons in lead-lead collisions at $\sqrt{s_{N N}}=2.76 \mathrm{TeV}$ with the ATLAS detector at the LHC, arXiv:1209.4910 [INSPIRE].

[11] CMS collaboration, Measurement of the differential cross section for isolated prompt photon production in pp collisions at 7 TeV, Phys. Rev. D 84 (2011) 052011 [arXiv:1108.2044] [INSPIRE].

[12] CMS collaboration, Measurement of isolated photon production in pp and $\mathrm{PbPb}$ collisions at $\sqrt{s_{N N}}=2.76 \mathrm{TeV}$, Phys. Lett. B 710 (2012) 256 [arXiv:1201.3093] [INSPIRE].

[13] CMS collaboration, Studies of jet quenching using isolated-photon+jet correlations in $\mathrm{PbPb}$ and pp collisions at $\sqrt{s_{N N}}=2.76 \mathrm{TeV}$, Phys. Lett. B 718 (2013) 773 [arXiv:1205.0206] [INSPIRE].

[14] D. d'Enterria and J. Rojo, Quantitative constraints on the gluon distribution function in the proton from collider isolated-photon data, Nucl. Phys. B 860 (2012) 311 [arXiv:1202.1762] [INSPIRE].

[15] L. Carminati et al., Sensitivity of the LHC isolated-gamma+jet data to the parton distribution functions of the proton, Europhys. Lett. 101 (2013) 61002 [arXiv:1212.5511] [INSPIRE].

[16] CDF collaboration, T. Aaltonen et al., Measurement of the cross section for prompt isolated diphoton production using the full CDF Run II data sample, Phys. Rev. Lett. 110 (2013) 101801 [arXiv:1212.4204] [INSPIRE].

[17] D0 collaboration, V. Abazov et al., Measurement of direct photon pair production cross sections in p pollisions at $\sqrt{s}=1.96$ TeV, Phys. Lett. B 690 (2010) 108 [arXiv: 1002.4917] [INSPIRE].

[18] D0 collaboration, V. Abazov et al., Measurement of the differential cross sections for isolated direct photon pair production in $p \bar{p}$ collisions at $\sqrt{s}=1.96 \mathrm{TeV}$, Phys. Lett. B 725 (2013) 6 [arXiv:1301.4536] [INSPIRE].

[19] ATLAS collaboration, Measurement of the isolated di-photon cross-section in pp collisions at $\sqrt{s}=7$ TeV with the ATLAS detector, Phys. Rev. D 85 (2012) 012003 [arXiv:1107.0581] [INSPIRE].

[20] ATLAS collaboration, Measurement of isolated-photon pair production in pp collisions at $\sqrt{s}=7 \mathrm{TeV}$ with the ATLAS detector, JHEP 01 (2013) 086 [arXiv:1211.1913] [INSPIRE]. 
[21] CMS collaboration, Measurement of the production cross section for pairs of isolated photons in pp collisions at $\sqrt{s}=7 \mathrm{TeV}$, JHEP 01 (2012) 133 [arXiv:1110.6461] [INSPIRE].

[22] CDF collaboration, T. Aaltonen et al., Search for anomalous production of events with two photons and additional energetic objects at CDF, Phys. Rev. D 82 (2010) 052005 [arXiv:0910.5170] [INSPIRE].

[23] D0 collaboration, V. Abazov et al., Search for large extra dimensions via single photon plus missing energy final states at $\sqrt{s}=1.96 \mathrm{TeV}$, Phys. Rev. Lett. 101 (2008) 011601 [arXiv:0803.2137] [INSPIRE].

[24] ATLAS collaboration, Search for dark matter candidates and large extra dimensions in events with a photon and missing transverse momentum in pp collision data at $\sqrt{s}=7 \mathrm{TeV}$ with the ATLAS detector, Phys. Rev. Lett. 110 (2013) 011802 [arXiv:1209.4625] [INSPIRE].

[25] CMS collaboration, Search for dark matter and large extra dimensions in pp collisions yielding a photon and missing transverse energy, Phys. Rev. Lett. 108 (2012) 261803 [arXiv: 1204.0821] [INSPIRE].

[26] CMS collaboration, Search for new physics in events with photons, jets and missing transverse energy in pp collisions at $\sqrt{s}=7 \mathrm{TeV}$, JHEP 03 (2013) 111 [arXiv:1211.4784] [INSPIRE].

[27] ATLAS collaboration, Search for the standard model Higgs boson in the diphoton decay channel with $4.9 \mathrm{fb}^{-1}$ of pp collisions at $\sqrt{\mathrm{s}}=7 \mathrm{TeV}$ with ATLAS, Phys. Rev. Lett. 108 (2012) 111803 [arXiv:1202.1414] [INSPIRE].

[28] ATLAS collaboration, Observation of a new particle in the search for the standard model Higgs boson with the ATLAS detector at the LHC, Phys. Lett. B 716 (2012) 1 [arXiv: 1207.7214] [INSPIRE].

[29] CMS collaboration, Search for the standard model Higgs boson decaying into two photons in pp collisions at $\sqrt{s}=7 \mathrm{TeV}$, Phys. Lett. B 710 (2012) 403 [arXiv:1202.1487] [INSPIRE].

[30] CMS collaboration, Observation of a new boson at a mass of $125 \mathrm{GeV}$ with the CMS experiment at the LHC, Phys. Lett. B 716 (2012) 30 [arXiv:1207.7235] [INSPIRE].

[31] M. Cacciari, G.P. Salam and G. Soyez, The catchment area of jets, JHEP 04 (2008) 005 [arXiv:0802.1188] [INSPIRE].

[32] M. Cacciari, G.P. Salam and S. Sapeta, On the characterisation of the underlying event, JHEP 04 (2010) 065 [arXiv:0912.4926] [INSPIRE].

[33] E.N. Glover and A. Morgan, Measuring the photon fragmentation function at LEP, Z. Phys. C 62 (1994) 311 [INSPIRE].

[34] A. Gehrmann-De Ridder, T. Gehrmann and E.N. Glover, Radiative corrections to the photon + 1 jet rate at LEP, Phys. Lett. B 414 (1997) 354 [hep-ph/9705305] [INSPIRE].

[35] S. Frixione, Isolated photons in perturbative QCD, Phys. Lett. B 429 (1998) 369 [hep-ph/9801442] [INSPIRE].

[36] J.R. Andersen et al., The SM and NLO multileg working group: summary report, arXiv: 1003.1241 [INSPIRE].

[37] J. Alcaraz Maestre et al., The SM and NLO Multileg and SM MC Working Groups: Summary Report, arXiv:1203.6803 [INSPIRE].

[38] S. Catani, M. Fontannaz, J. Guillet and E. Pilon, Cross-section of isolated prompt photons in hadron hadron collisions, JHEP 05 (2002) 028 [hep-ph/0204023] [INSPIRE]; JETPHOX is available at http://lapth.in2p3.fr/PHOX_FAMILY/main.html. 
[39] T. Binoth, J. Guillet, E. Pilon and M. Werlen, A full next-to-leading order study of direct photon pair production in hadronic collisions, Eur. Phys. J. C 16 (2000) 311 [hep-ph/9911340] [INSPIRE]; DIPHOX is available at http://lapth.in2p3.fr/PHOX_FAMILY/main.html.

[40] L. Gordon and W. Vogelsang, Polarized and unpolarized prompt photon production beyond the leading order, Phys. Rev. D 48 (1993) 3136 [INSPIRE].

[41] J.M. Campbell, R.K. Ellis and C. Williams, Vector boson pair production at the LHC, JHEP 07 (2011) 018 [arXiv:1105.0020] [INSPIRE].

[42] Z. Bern, L.J. Dixon and C. Schmidt, Isolating a light Higgs boson from the diphoton background at the CERN LHC, Phys. Rev. D 66 (2002) 074018 [hep-ph/0206194] [INSPIRE].

[43] S. Catani, L. Cieri, D. de Florian, G. Ferrera and M. Grazzini, Diphoton production at hadron colliders: a fully-differential QCD calculation at NNLO, Phys. Rev. Lett. 108 (2012) 072001 [arXiv: 1110.2375] [INSPIRE].

[44] Z. Belghobsi et al., Photon-jet correlations and constraints on fragmentation functions, Phys. Rev. D 79 (2009) 114024 [arXiv:0903.4834] [INSPIRE].

[45] V. Del Duca, F. Maltoni, Z. Nagy and Z. Trócsányi, QCD radiative corrections to prompt diphoton production in association with a jet at hadron colliders, JHEP 04 (2003) 059 [hep-ph/0303012] [INSPIRE].

[46] Z. Bern et al., Driving missing data at next-to-leading order, Phys. Rev. D 84 (2011) 114002 [arXiv: 1106.1423] [INSPIRE].

[47] T. Gehrmann, N. Greiner and G. Heinrich, Photon isolation effects at NLO in $\gamma \gamma+$ jet final states in hadronic collisions, arXiv:1303.0824 [INSPIRE].

[48] C. Balázs, E.L. Berger, P.M. Nadolsky and C.-P. Yuan, Calculation of prompt diphoton production cross-sections at Tevatron and LHC energies, Phys. Rev. D 76 (2007) 013009 [arXiv: 0704.0001] [INSPIRE].

[49] T. Becher and M.D. Schwartz, Direct photon production with effective field theory, JHEP 02 (2010) 040 [arXiv: 0911.0681] [INSPIRE].

[50] T. Becher, C. Lorentzen and M.D. Schwartz, Precision direct photon and W-boson spectra at high $p_{T}$ and comparison to LHC data, Phys. Rev. D 86 (2012) 054026 [arXiv:1206.6115] [INSPIRE].

[51] S.P. Baranov, A.V. Lipatov and N.P. Zotov, Prompt photon hadroproduction at high energies in off-shell gluon-gluon fusion, Phys. Rev. D 77 (2008) 074024 [arXiv:0708.3560] [INSPIRE].

[52] A.V. Lipatov, M.A. Malyshev and N.P. Zotov, Testing for $k_{t}$-factorization with inclusive prompt photon production at LHC, Phys. Lett. B 699 (2011) 93 [arXiv:1102.1134] [INSPIRE].

[53] G. Diana, J. Rojo and R.D. Ball, High energy resummation of direct photon production at hadronic colliders, Phys. Lett. B 693 (2010) 430 [arXiv:1006.4250] [INSPIRE].

[54] S. Hoeche, S. Schumann and F. Siegert, Hard photon production and matrix-element parton-shower merging, Phys. Rev. D 81 (2010) 034026 [arXiv:0912.3501] [INSPIRE].

[55] L. D'Errico and P. Richardson, Next-to-leading-order Monte Carlo simulation of diphoton production in hadronic collisions, JHEP 02 (2012) 130 [arXiv:1106.3939] [INSPIRE]. 
[56] S. Odaka and Y. Kurihara, Consistent simulation of non-resonant diphoton production at hadron collisions with a custom-made parton shower, Phys. Rev. D 85 (2012) 114022 [arXiv:1203.4038] [INSPIRE].

[57] L. Bourhis, M. Fontannaz and J. Guillet, Quarks and gluon fragmentation functions into photons, Eur. Phys. J. C 2 (1998) 529 [hep-ph/9704447] [INSPIRE].

[58] P. Aurenche, M. Fontannaz and J.P. Guillet, New NLO parametrizations of the parton distributions in real photons, Eur. Phys. J. C 44 (2005) 395 [hep-ph/0503259] [INSPIRE].

[59] CTEQ collaboration, J. Pumplin et al., New generation of parton distributions with uncertainties from global QCD analysis, JHEP 07 (2002) 012 [hep-ph/0201195] [INSPIRE]. 